\title{
Reduction of Molecular Leaching from a Gel Matrix for the Precisely Controlled Release of Encapsulated Molecules by Light Stimulus
}

\author{
Takaki Amamoto, Tomofumi Santa, and Masaru Kato* \\ Graduate School of Pharmaceutical Sciences and GPLLI Program, The University of Tokyo; 7-3-1 Hongo, \\ Bunkyo-ku, Tokyo 113-0033, Japan. \\ Received January 28, 2014; accepted April 17, 2014
}

\begin{abstract}
We have developed a general method for controlling molecular functions using a photodegradable hydrogel; gels containing molecules made from such materials are capable of release and activation by light stimulus. As the elimination of molecular leaching from the gel before irradiation was a barrier to the precise control of molecular function, we optimized the monomer used in gel preparation during this study. The addition of $N, N^{\prime}$-methylenebis(acrylamide) (MBAA) inhibited molecular leaching from the gel; the MBAA concentration is a critical factor in controlling the leaching of encapsulated molecules. We succeeded in preparing a gel that halved the leaching of small encapsulated molecules, while the leaching of large molecules, such as albumin $(66 \mathrm{kDa})$ and ferritin $(450 \mathrm{kDa})$, was at negligible levels, or disappeared. The on/off ratios (released amount/leached amount) of albumin and ferritin were 8 and 17, respectively.
\end{abstract}

Key words protein; spatiotemporal control; photo induce; gel; leach; mesh structure

The immobilization of functional molecules in matrices is an effective way of utilizing such molecules, because it enables the addition of specific molecules with defined properties according to their intended purpose, while allowing their removal after use ${ }^{1-4)}$; immobilized functional molecules are finding ever increased use in a variety of applications from analytical chemistry to manufacturing and environmental remediation. ${ }^{5-7)}$

We previously reported a photodegradable hydrogel that encapsulated functional molecules within the mesh structure of the hydrogel; the encapsulated molecules are subsequently released on gel degradation, induced by light stimulus. ${ }^{8)} \mathrm{Be}$ cause the gel can be used as a photocontrol method for the activity of various molecules, we have employed a method, which we refer to as PARCEL (Protein Activation and Release from Cage by External Light) methods, ${ }^{9-12)}$ for the spatiotemporal activation of molecules such as proteins, small molecules, and small interfering RNA (siRNA) within cells. ${ }^{13)}$

The reduction or disappearance of molecular leaching prior to the application of a stimulus is one of the critical remaining issues to be overcome in this area because molecules that are leached prior to the application of a stimulus show activity after irradiation, making the precise control of molecular functions difficult; many studies have been carried out on the reduction of leaching from the immobilizing matrix. ${ }^{14-16)}$

Leaching originates in the release of molecules from gel matrices (without stimulus) where molecules are not firmly immobilized, which cannot be removed by washing. We envisaged that molecular leaching would be decreased by reducing the mesh size of the gel, leaving the encapsulated molecule trapped in the mesh structure of the gel. We have succeeded in reducing the leaching of small molecules by reducing the mesh size of the gel through reduction of the monomer size used in the preparation of the gel. ${ }^{9)}$

In this study, we performed detailed investigations into the effect of the mesh structure on molecular leaching from the gel. Two different types of monomer were used for the preparation of the photodegradable gel: a linear monomer,

The authors declare no conflict of interest such as acrylamide (AA), that forms linear polymers after polymerization, and a branched monomer containing four-armed polyethylene $^{11)}$ or polyamine moieties ${ }^{10)}$ that form branched structures within the gel. Two modes of cross-linking were observed in the mesh structure of the gel: physical crosslinking and chemical cross-linking. ${ }^{17,18)}$ Physical cross-linking occurs via the intertwining of linear polymers, whereas chemical cross-linking occurs through the branched structure of the polymer. To reduce the mesh size of the gel prepared via physical cross-linking, it was necessary to increase the concentration of the linear polymer. To reduce the mesh size of the gel prepared by chemical cross-linking, it was necessary to increase the number of branched structures. Hence, the amount of physical cross-linking was mainly determined by the amount of linear monomer present; the amount of chemical cross-linking was similarly determined by the amount of branched monomer. We, therefore, prepared gels of different monomer compositions, and examined the effect of the gel component on molecular leaching from the gel. We also used $N, N^{\prime}$-methylenebis(acrylamide) (MBAA) for the introduction of chemical cross-linking in the gel, as MBAA is often used to control the mesh size in gels. ${ }^{19)}$

\section{Experimental}

Materials and Chemicals Tetra-poly(ethyl glycol)-amine (SUNBRIGHT PTE-050PA; Mn, $5328 \mathrm{~g} / \mathrm{mol}$ ) was purchased from NOF Corporation (Tokyo, Japan). $N, N, N^{\prime}, N^{\prime}$-Tetrameth ylethylenediamine (TEMED), dichloromethane (DCM), acryloyl chloride (AC), triethylamine (TEA), ammonium persulfate (APS), acrylamide (AA), $N, N^{\prime}$-methylene-bis(acrylamide) (MBAA), tris(hydroxymethyl) aminomethane, hydrochloric acid, methanol, acetone, 1-butyl alcohols, diethyl ether, trypsin, bovine serum albumin, and 4-(4,6-dimethoxy-1,3,5triazin-2-yl)-4-methylmorpholinium chloride $n$-hydrate (DMT$\mathrm{MM})$ were purchased from Wako Pure Chemical Industries, Ltd. (Osaka, Japan). 4-[4-(1-Hydroxyethyl)-2-methoxy-5nitrophenyl]butyric acid, fluorescein isothiocyanate-dextran (Flu-dex, molecular weight $(\mathrm{MW})=20000$ and 4000), and ferritin were purchased from Sigma-Aldrich (St. Louis, MO, U.S.A.). Flu-dex $(\mathrm{MW}=40000)$ was obtained from Life Tech- 
nologies Corporation (Carlsbad, CA, U.S.A.). Pierce bicinchoninic acid (BCA) protein assay reagent $\mathrm{A}$ and $\mathrm{B}$ were from Thermo Fisher Scientific Inc. (Rockford, IL, U.S.A.). Water was purified with a Milli-Q apparatus (Millipore, Bedford, MA, U.S.A.).

PEG-Photo-Ac Synthesis A procedure, similar to one reported previously, ${ }^{10}$ ) was used for the synthesis of PEGPhoto-Ac. The photolabile molecule 4-[4-(1-hydroxyethyl)-2methoxy-5-nitro-phenoxy]butyric acid $(1 \mathrm{mmol})$ was dissolved in dry DCM and stirred in an amber glass vial purged with $\mathrm{N}_{2}$ gas. TEA $(3 \mathrm{mmol})$ and $\mathrm{AC}(2.5 \mathrm{mmol})$, both dissolved in dry DCM, were added dropwise at $0^{\circ} \mathrm{C}$. The reaction was stirred at room temperature overnight. The reaction solution was washed with sodium bicarbonate $(5 \mathrm{w} / \mathrm{v} \%$ aq.), dilute hydrochloric acid ( $1 \mathrm{v} / \mathrm{v} \%$ aq.), and water. The solution was evaporated, and the liquid product was dissolved in aqueous acetone $(50 \mathrm{v} / \mathrm{v} \%$ aq.). This reaction mixture was stirred at room temperature overnight and then extracted with DCM to recover the liquid acrylated monomer. The DCM layer was washed with dilute hydrochloric acid ( $1 \mathrm{v} / \mathrm{v} \%$ aq.) and water, dried over magnesium sulfate, and evaporated to yield the photocleavable acrylate monomer referred to as Photo-Ac.

Photo-Ac $(5 \mathrm{mmol})$ was dissolved in methanol and stirred. Then, tetra poly(ethylene glycol)-amine (tetra-PEG-amine; $1 \mathrm{mmol}$ ) was added and stirred until all reactants were dissolved. After the addition of DMT-MM, the stirring was stopped. The reaction was carried out at room temperature and allowed to run overnight. The product was precipitated in diethyl ether on ice and filtered. The collected substance was washed with diethyl ether and dissolved in water. The aqueous solution was dialyzed (SpectraPor6, CO $1000 \mathrm{~g} / \mathrm{mol}$ ) and freeze-dried to yield the tetra-acrylated PEG, referred to as PEG-Photo-Ac (Fig. 1).

Polymerization Reaction for the Preparation of the Gels
Containing Molecules The gels containing molecules were prepared in 96-well micro assay plates (BD, Franklin Lakes, NJ, U.S.A.). Solutions of $100 \mathrm{mg} / \mathrm{mL}$ PEG-Photo-Ac $(100 \mu \mathrm{L})$, a mixture of $380 \mathrm{mg} / \mathrm{mL}$ AA and $20 \mathrm{mg} / \mathrm{mL}$ MBAA $(100 \mu \mathrm{L})$, the encapsulated molecule $(0.25 \mathrm{mg} / \mathrm{mL}$ Flu-dex or $10 \mathrm{mg} / \mathrm{mL}$ protein, $100 \mu \mathrm{L}), 0.1 \mathrm{M}$ APS $(50 \mu \mathrm{L})$, and $0.1 \mathrm{M}$ TEMED $(50 \mu \mathrm{L})$ were dissolved in water in that order; $50 \mu \mathrm{L}$ of the mixture was dispensed into the wells of the micro assay plate. The gelation reaction was carried out in the plate at $37^{\circ} \mathrm{C}$ for $2 \mathrm{~h}$, and each well was washed with water until the fluorescence intensity of the washed solution became constant (approximately 10 washes). A schematic diagram of the gel is shown in Fig. 1.

Gel Irradiation An Aicure UV20 (Panasonic, Osaka, Japan) UV curing apparatus was used as a UV irradiation light source; the wavelength of the irradiating light was $365 \mathrm{~nm}$ at an intensity of $20 \mathrm{~mW} / \mathrm{cm}^{2}$. Half of the gel was irradiated with UV light for $30 \mathrm{~s}$; the remaining half of the gel was not subjected to irradiation.

Measurement of Flu-Dex Leaching and Release from the Gels After irradiation, the gels were incubated for $3 \mathrm{~min}$ with shaking, before extracting $100 \mu \mathrm{L}$ of the upper layer contained in the well. The fluorescence (excitation $494 \mathrm{~nm}$, emission $521 \mathrm{~nm}$ ) of the released Flu-dex was measured in the extract with a multiplate reader (SH-9000, Corona Electric Co., Ibaraki, Japan). The same procedure was used to measure the amount of leaching from a sample solution obtained from a well before irradiation. The leached and released amounts were defined as the number of molecules present in the supernatant before (leached amount) and after irradiation (released amount), respectively.

Measurement of the Amount of Protein Leaching and Release from the Gel The BCA protein assay method was used to measure the protein content in the upper layer of the wells in the 96-well micro assay plate. The sample solution

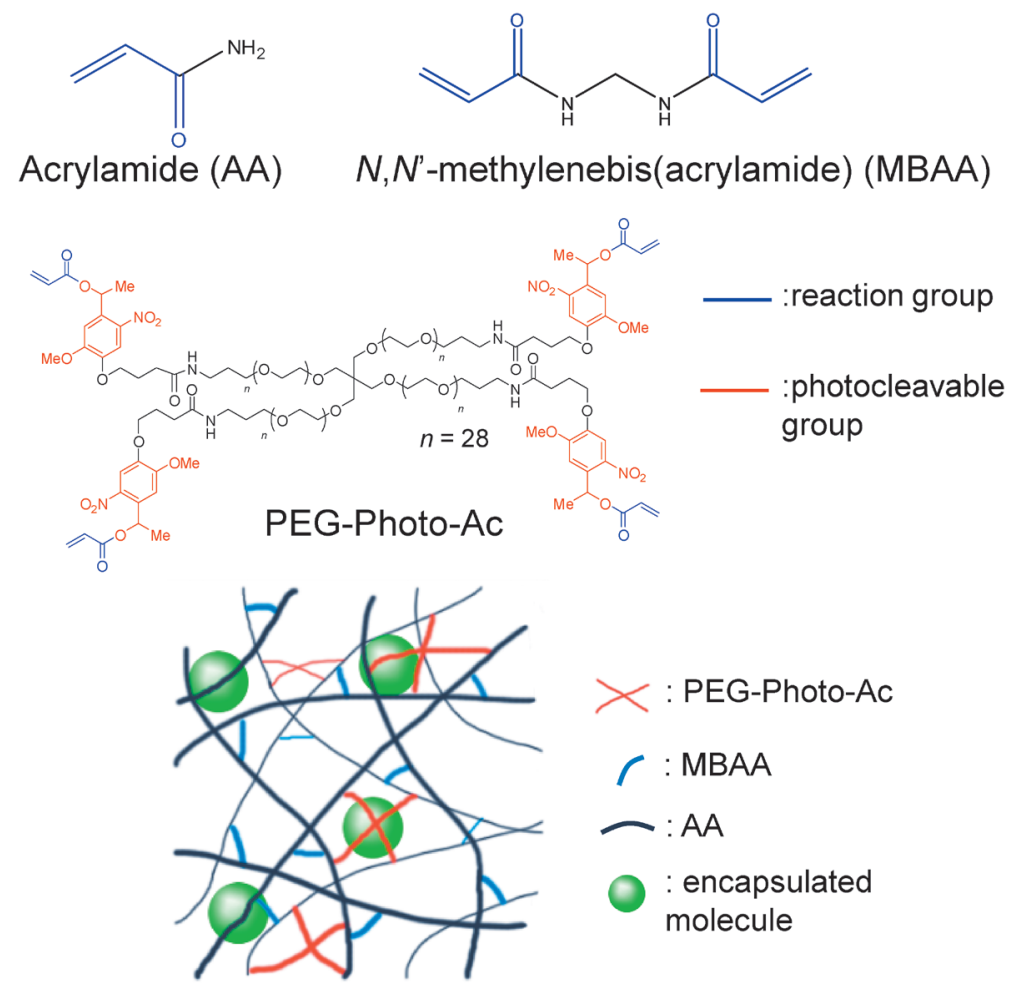

Fig. 1. Structures of the Monomers Used for the Photodegradable Gel and a Schematic Image of the Gel 
$(25 \mu \mathrm{L})$ and the BCA reagent (a mixture of Pierce BCA protein assay reagents $\mathrm{A}$ and $\mathrm{B}$ in a $50: 1$ ratio, $200 \mu \mathrm{L}$ ) were dispensed into the well and mixed thoroughly on a plate shaker for $30 \mathrm{~s}$. The plate was incubated at $37^{\circ} \mathrm{C}$ for $30 \mathrm{~min}$ and cooled to room temperature. The absorbance at $562 \mathrm{~nm}$ was measured by a multiplate reader.

\section{Results and Discussion}

Effect of AA Concentration Because the mesh size of the gel was tunable by the AA concentration, we studied the effect of AA concentration (from 100 to $200 \mathrm{mg} / \mathrm{mL}$ ) on the leaching of encapsulated molecules and release after irradiation. The amount of physical cross-linking in the gel increased with increasing AA concentration, decreasing the mesh size of the gel. Figure 2 shows the leaching and released amounts of Flu-dex $(\mathrm{MW}=20 \mathrm{kDa})$ from the gel; we found that the amount of molecular leaching from the gel decreased with increasing AA concentration. However, an increase in the AA concentration not only decreased the leaching of encapsulated molecules, but also decreased the molecular release after irradiation. This is because increasing the AA concentration decreased the mesh size of the gel, ${ }^{19)}$ thereby decreasing both the leaching and the release of encapsulated molecules from the gel. The release of molecules from the mesh structure of the gel requires appropriately sized gaps; it was difficult to form such gaps at high AA concentrations, because numerous cleavage reactions were required. This result indicated that the AA concentration is not a suitable factor for reducing molecular leaching from the gel.

Effect of MBAA Concentration Because AA forms physical cross-links (Fig. 1), a gel prepared solely from AA is a physical gel, i.e., the mesh structure of the gel is formed by the intertwining of linear polymers. We envisaged that the gel properties would change upon adding MBAA to the solution used in gel preparation because, in addition to physical cross-linking, chemical cross-linking is introduced in the mesh structure of the gel (Fig. 1). We determined the amount of leaching and release of Flu-dex $(\mathrm{MW}=20 \mathrm{kDa})$ from the gel in the MBAA concentration range of $0-11 \mathrm{mg} / \mathrm{mL}$ (Fig. 3). Leaching from gels prepared with MBAA was significantly lower than that from gels prepared without MBAA. The leaching reached a minimum when the MBAA concentration was

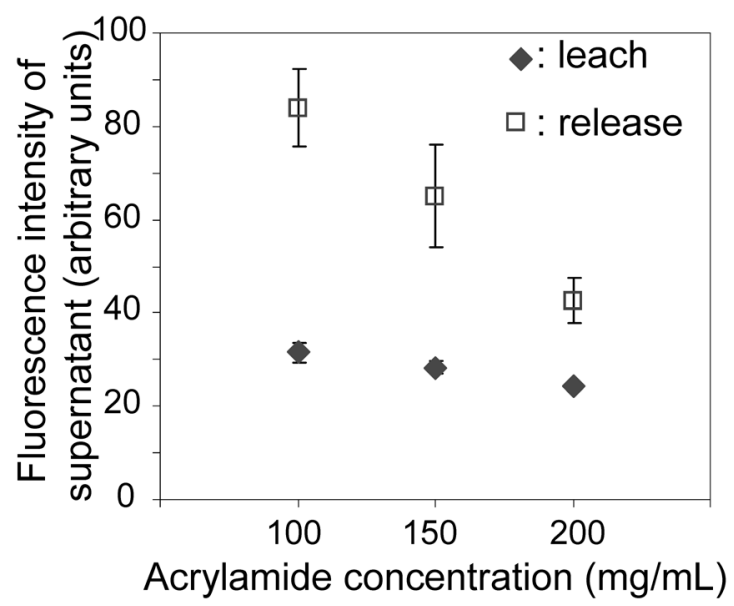

Fig. 2. Effect of Acrylamide (AA) Concentration on the Leaching and Release of Flu-Dex from the Gel
$>5 \mathrm{mg} / \mathrm{mL}$. We observed a small decrease in the amount of released molecules when the MBAA concentration was increased; however, the decrease was less than that with AA. Therefore, the addition of MBAA is an effective way to reduce molecular leaching from a gel. We therefore chose $5 \mathrm{mg}$ / mL MBAA as an optimum concentration.

Effect of PEG-Photo-Ac Concentration Because PEGPhoto-Ac contains four reactive groups, it also forms chemical cross-links in a manner similar to that of MBAA. Figure 4 shows the leaching and released amounts of Flu-dex (MW= $20 \mathrm{kDa}$ ) from the gel. The amount of leached molecules slightly decreased with increasing PEG-Photo-Ac concentrations in a PEG-Photo-Ac concentration range of $12.5-50 \mathrm{mg} / \mathrm{mL}$. However, the decrease reached a plateau at $25 \mathrm{mg} / \mathrm{mL}$ PEGPhoto-Ac, and the reproducibility of the experimentally measured amount of leached molecules was low when the gel was prepared from $>37.5 \mathrm{mg} / \mathrm{mL}$ PEG-Photo-Ac. PEG-Photo-Ac is a high-molecular-weight compound with four acryloyl groups and long PEG chains (28 repeat units) in the molecule, and PEG-Photo-Ac is incorporated into the gel by the reaction of one of the acryloyl groups with the mesh structure of the gel. We believe that the flexibility of the gel is increased by the incorporation of PEG-Photo-Ac, because some of the acryloyl groups of PEG-Photo-Ac remain in unreacted form after the

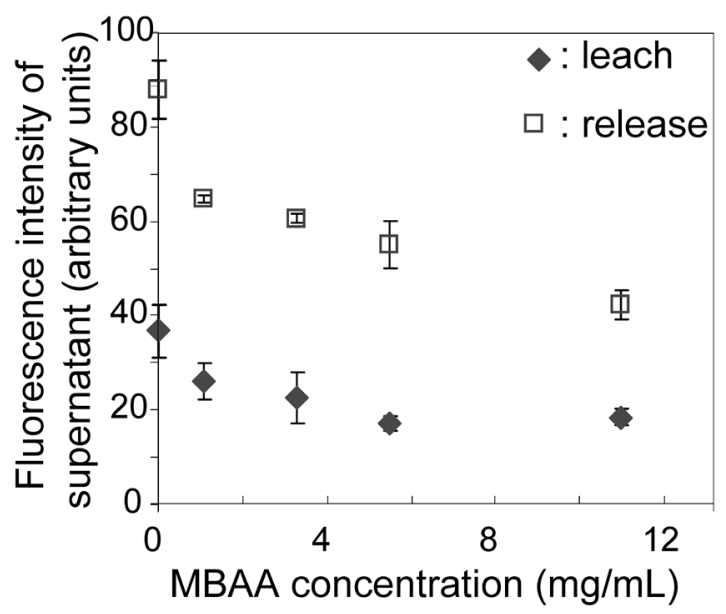

Fig. 3. Effect of $N, N^{\prime}$-Methylene-bis(acrylamide) (MBAA) Concentration on the Leaching and Release of Flu-Dex from the Gel

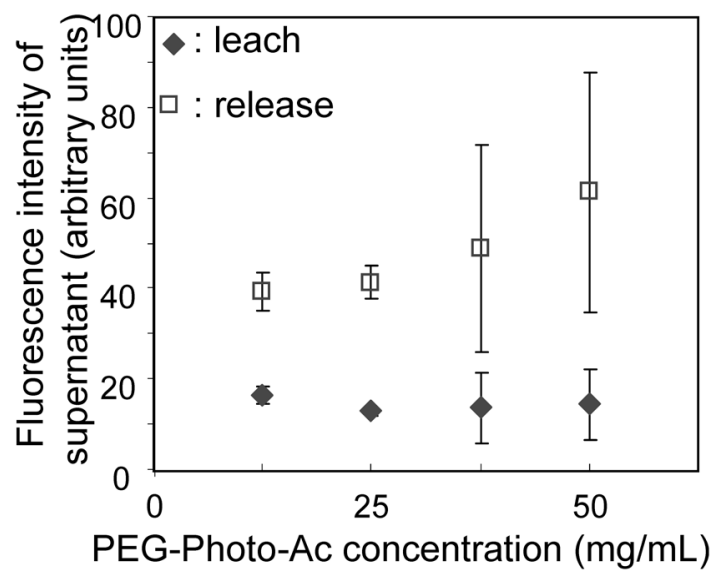

Fig. 4. Effects of PEG-Photo-Ac Concentration on the Leaching and Release of Flu-Dex from the Gel 
reaction, and PEG-Photo-Ac possesses long PEG chains. ${ }^{20)} \mathrm{We}$ used a PEG-Photo-Ac concentration of $25 \mathrm{mg} / \mathrm{mL}$ for further investigations.

Comparison of the Leached Amount and the On/Off Ratio of Encapsulated Molecules The amount of molecular leaching was measured using the optimized gel. Three dextran derivatives $(4,20,40 \mathrm{kDa})$ and proteins (trypsin, albumin, and ferritin) with different molecular weights were used as the encapsulated molecules. Figure 5 shows the amount of leaching from the gel before irradiation. The amount of leaching decreased with increasing molecular weight of the encapsulated molecule. The leached amounts of albumin and ferritin were lower than the detection limit, meaning that the leaching of these proteins either disappeared entirely or occurred at a negligible level. Figure 1 in the Supporting Information shows the released amount of dextran derivatives, and the result indicated that the released amount decreased with increasing molecular weight of the dextran.

We compared the on/off ratio of each encapsulated molecule, which was calculated from the following equation:

on/off ratio $=$ released amount $/$ leached amount

Table 1 summarizes the on/off ratio for each molecule. The on/off ratio of dextran was constant at 1.3 regardless of the dextran molecular weight. This was because the released amount decreased in accordance with the decrease in the leached amount (Supporting Fig. 1). The protein on/off ratio was larger than that of the dextran. We thought that the degradation reaction that the encapsulated molecule undergoes on irradiation was negligible, because the on/off ratio of trypsin, measured by enzyme activity and the BCA method were similar (data not shown). The values of the ratio for large proteins

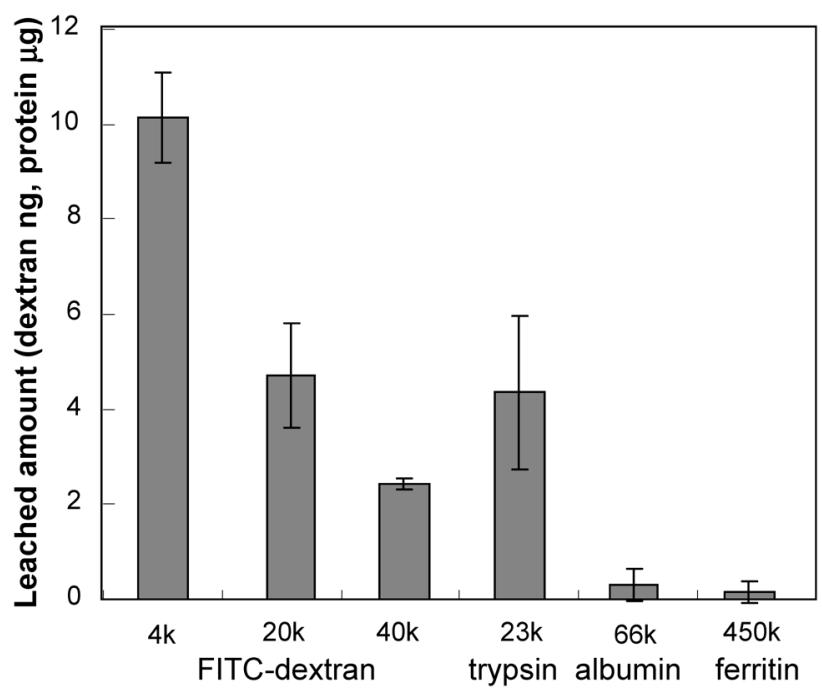

Fig. 5. Effect of the Encapsulated Molecules on the Leaching Amounts of Each Molecule (albumin and ferritin) that leached-effectively hindered by the optimized gel-were large; therefore, minimizing leaching is important for improving the on/off ratio. Figure 2 in the Supporting Information showed the relationship between the incubation time and released amount of Flu-dex $(20 \mathrm{kDa})$. It can be seen that the released amount of Flu-dex increased with incubation time. We believe that the optimized gels, which exhibit minimal molecular leaching, developed in this study are excellent candidates for controlling the activity of encapsulated large proteins.

In our previous study, ${ }^{9}$ we prepared a photodegradable gel with a small mesh size by reducing the size of the branched monomer. We successfully used this gel to reduce molecular leaching from the gel before irradiation. However, the released amount of the encapsulated molecule after irradiation also decreased, and the released amount of large molecules $(70 \mathrm{kDa}$ Flu-dex) was very small. Therefore, MBAA, which possesses two reactive groups at both ends of the molecule, was used to reduce the gel mesh size in this study. The gel developed in this study also exhibits decreased molecular leaching, but can release the encapsulated molecules efficiently on irradiation, even when the molecular size was $c a .450 \mathrm{kDa}$. Although four-armed monomers form a mesh structure by forming covalent bonds with neighboring four-armed monomers or linear polymers, MBAA forms a mesh structure by connecting two linear polymers. Therefore, we suggest that the size of mesh structures formed by MBAA were more heterogeneous and larger than those formed by four-armed monomers; this size difference between the mesh structures causes the different release properties of the encapsulated molecules. Further studies are needed to clarify the relationship between the mesh structure of the gel and the release properties of the encapsulated molecules.

\section{Conclusion}

In this study, we tried to eliminate molecular leaching from photodegradable gels before the application of a stimulus by reducing the mesh size of the gel. The amount of leaching was controlled by the MBAA concentration; MBAA inhibited leaching without decreasing the repeatability of the experimental measurements.

As we successfully prepared a gel that can encapsulate functional molecules with little or no leaching before irradiation, we expect that such gels will be applicable in accurate optical activation sensors (no signal before optical activation) or safe drug-delivery systems (no leaching before arrival at the target tissue).

Acknowledgments This work was supported by Grants (Kakenhi) from the Ministry of Education, Culture, Sports, Science and Technology (MEXT) of Japan, JSPS Core-toCore Program, A. Advanced Research Networks, and the Naito Foundation.

Table 1. Comparison of on/off Ratio of Flu-Dex and Proteins

\begin{tabular}{|c|c|c|c|c|c|c|}
\hline & \multicolumn{3}{|c|}{ Flu-dex } & \multirow{2}{*}{ Trypsin (23 kDa) } & \multirow{2}{*}{ Albumin $(66 \mathrm{kDa})$} & \multirow{2}{*}{ Ferritin $(450 \mathrm{kDa})$} \\
\hline & $(4 \mathrm{kDa})$ & $(20 \mathrm{kDa})$ & $(40 \mathrm{kDa})$ & & & \\
\hline On/off ratio & 1.3 & 1.3 & 1.3 & 3.1 & 8.0 & 17 \\
\hline
\end{tabular}




\section{References}

1) Zhou Z., Hartmann M., Chem. Soc. Rev., 42, 3894-3912 (2013).

2) Hartmann M., Chem. Mater., 17, 4577-4593 (2005).

3) Chaki N. K., Vijayamohanan K., Biosens. Bioelectron., 17, 1-12 (2002).

4) Kato M., Sakai-Kato K., Toyo'oka T., Anal. Bioanal. Chem., 384, 50-52 (2006)

5) Kato M., Sakai-Kato K., Matsumoto N., Toyo'oka T., Anal. Chem., 74, 1915-1921 (2002).

6) Sakai-Kato K., Kato M., Ishihara K., Toyo'oka T., Lab Chip, 4, 4-6 (2004).

7) Kato M., Sakai-Kato K., Jin H.-M., Kubota K., Miyano H., Toyo'oka T., Dulay M. T., Zare R. N., Anal. Chem., 76, 1896-1902 (2004).

8) Murayama S., Kato M., Anal. Chem., 82, 2186-2191 (2010).

9) Murayama S., Ishizuka F., Takagi K., Inoda H., Sano A., Santa T., Kato M., Anal. Chem., 84, 1374-1379 (2012).

10) Murayama S., Nishiyama T., Takagi K., Ishizuka F., Santa T., Kato M., Chem. Commun. (Camb.), 48, 11461-11463 (2012).
11) Murayama S., Su B., Okabe K., Kishimura A., Osada K., Miura M., Funatsu T., Kataoka K., Kato M., Chem. Commun. (Camb.), 48, 8380-8382 (2012).

12) Murayama S., Jo J., Shibata Y., Liang K., Santa T., Saga T., Aoki I., Kato M., J. Mater. Chem. B, 1, 4932-4938 (2013).

13) Murayama S., Kos P., Miyata K., Kataoka K., Wagner E., Kato M., Macromol. Biosci., 14, 626-631 (2014).

14) Díaz J. F., Balkus K. J. Jr., J. Mol. Catal., B Enzym., 2, 115-126 (1996).

15) Sörensen M. H., Ng J. B. S., Bergström L., Alberius P. C. A., J. Colloid Interface Sci., 343, 359-365 (2010).

16) Wang Y., Caruso F., Chem. Mater., 17, 953-961 (2005).

17) Sangeetha N. M., Maitra U., Chem. Soc. Rev., 34, 821-836 (2005).

18) Kato M., Shoda N., Yamamoto T., Shiratori R., Toyo'oka T., Analyst (Lond.), 134, 577-581 (2009).

19) Rüche R., Steere R. L., Erbe E. F., J. Chromatogr. A, 166, 563-575 (1978).

20) Takagi K., Murayama S., Sakai T., Asai M., Santa T., Kato M., Soft Matter, 10, 3553-3559 (2014). 